\title{
Stability of Polar Oxide Surfaces
}

\author{
A. Wander, ${ }^{1}$ F. Schedin, ${ }^{2}$ P. Steadman, ${ }^{3}$ A. Norris, ${ }^{4}$ R. McGrath, ${ }^{4}$ T. S. Turner, ${ }^{1}$ G. Thornton, ${ }^{2}$ and N. M. Harrison ${ }^{1,5}$ \\ ${ }^{1}$ CLRC, Daresbury Laboratory, Daresbury, Warrington, WA4 4AD, United Kingdom \\ ${ }^{2}$ Surface Science Centre and Chemistry Department, Manchester University, Manchester M13 9PL, United Kingdom \\ ${ }^{3}$ ESRF, B.P. 220, F-38043 Grenoble Cedex, France \\ ${ }^{4}$ Surface Science Centre, Liverpool University, Liverpool L69 3BX, United Kingdom \\ ${ }^{5}$ Department of Chemistry, Imperial College of Science and Technology, London, SW7 2AY, United Kingdom
}

(Received 7 August 2000)

\begin{abstract}
The structures of the polar surfaces of $\mathrm{ZnO}$ are studied using ab initio calculations and surface $\mathrm{x}$-ray diffraction. The experimental and theoretical relaxations are in good agreement. The polar surfaces are shown to be very stable; the cleavage energy for the (0001)- $\mathrm{Zn}$ and (0001)-O surfaces is $4.0 \mathrm{~J} / \mathrm{m}^{2}$ comparable to $2.32 \mathrm{~J} / \mathrm{m}^{2}$ for the most stable nonpolar (1010) surface. The surfaces are stabilized by an electronic mechanism involving the transfer of 0.17 electrons between them. This leads to 2D metallic surface states, which has implications for the use of the material in gas sensing and catalytic applications.
\end{abstract}

DOI: $10.1103 /$ PhysRevLett.86.3811

PACS numbers: 68.03.Cd, 68.35.Bs, 73.20.At

The ionic model has provided the basis for our understanding of the very wide range of physical phenomena displayed by "ionic" crystals [1-4]. The model underpins our understanding of, for instance; cohesive properties, complex dielectric and optical response, and novel magnetic and electronic behavior including giant magnetoresistance and superconductivity [2]. One of the interesting consequences of the ionic model is that certain "polar" surfaces of ionic crystals will have a surface energy that diverges with sample size due to the generation of a macroscopic electrostatic field across the crystal. A definitive description of this behavior and the classification scheme which is now widely used were given by Tasker in 1979 [5]. Remarkably, a large number of naturally occurring materials have morphologies which display polar surfaces. In recent years a variety of stabilizing mechanisms have been demonstrated to operate at particular surfaces which typically involve the quenching of the macroscopic field either through the reconstruction of the surface, or the presence of adsorbates on the surface [3]. However, in some cases it appears that clean, unreconstructed surfaces are stable, at variance with Tasker's conclusions. A notable example is zincite $(\mathrm{ZnO})$. A possible mechanism for the stability of these systems is a rearrangement of the electronic structure resulting in an effective charge transfer between the polar surfaces removing the macroscopic field which would otherwise be present. To date, no first principles investigation of such a phenomenon has been performed, although semiempirical calculations on $\mathrm{SrTiO}_{3}$ indicate that such a mechanism might be operating [6,7]. Recently, similar effects have also been observed for thin film ionic materials grown on metallic substrates such as $\mathrm{NaCl}(111)$ on aluminum [8].

$\mathrm{ZnO}$ crystallizes in the Wurtzite structure which does not have a center of inversion. Consequently, when the crystal is cleaved normal to the $c$ axis in a manner which breaks the fewest interatomic bonds, two different polar surfaces are formed on opposite sides of the crystal, each having only one type of ion in its outermost plane. Thus, such a system may be considered to be a "slab" of material with the Zn cation outermost for the (0001)-Zn surface and

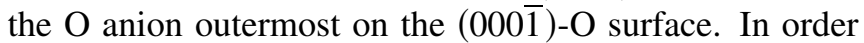
to conserve stoichiometry and ensure charge neutrality of the crystal, any slab which has a (0001)-Zn surface on one side must have a $(000 \overline{1})-O$ surface on the other side, as illustrated in Fig. 1. Within the ionic model it is clear that this slab generates a macroscopic field perpendicular to the surface and that its energy diverges with slab thickness. Our surface $\mathrm{x}$-ray diffraction data clearly demonstrate that the $(000 \overline{1})-\mathrm{O}$ surface of $\mathrm{ZnO}$ is unreconstructed. This is supported by scanning tunneling microscopy images of the same surface which show no defects other than steps on the surface [9].

In the current study the mechanism behind the stabilization of these surfaces has been investigated by a combination of $a b$ initio density functional calculations, and surface $\mathrm{x}$-ray diffraction experiments. This approach reveals, unambiguously, that the surface is stabilized by a rearrangement of the electronic structure of both the polar surfaces. The density functional calculations were performed utilizing a hybrid functional to treat electron exchange and correlation interactions (the B3LYP functional [10,11]) implemented within a linear combination of atomic orbitals framework [12]. The B3LYP functional is known to model accurately the energetics, geometry, and electronic properties of oxide materials [13]. It has previously been shown to provide an excellent description of both bulk $\mathrm{ZnO}$ and its nonpolar (1010) surface [14].

Previous attempts to model polar surfaces from first principles have artificially removed the macroscopic field from the slab [15]. In contrast to these studies we have performed calculations on slabs periodic in two dimensions but finite in the third (such as that illustrated in Fig. 1). Perpendicular to the surface the boundary condition that 


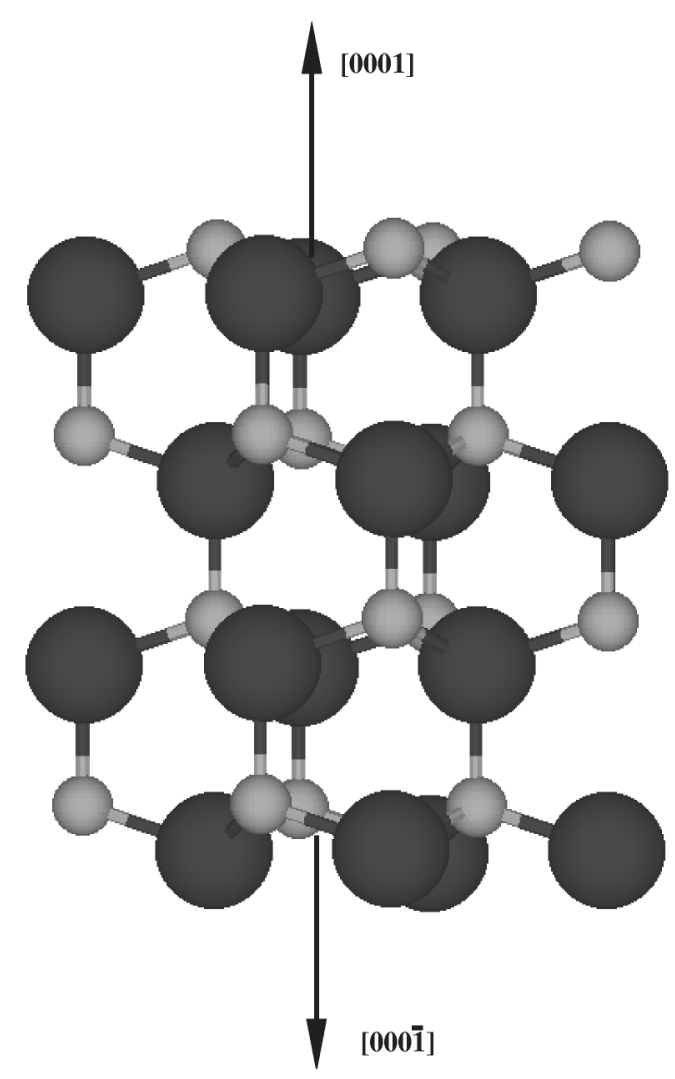

FIG. 1. A side view of the polar (0001) and (0001) $\mathrm{ZnO}$ surfaces.

the wave function decays to zero at infinity is applied. As the slab can support an electric field, the convergence of the surface energy with respect to slab thickness can be achieved only (in the absence of other mechanisms) if the electronic structure generates a field which converges to a finite value.

The slab thickness is referenced by the number of bilayers it contains, and hence Fig. 1 is referred to as an S4 slab. Calculations relaxing both the electronic and geometric degrees of freedom were performed on slabs of increasing thickness. The relaxation of the surface geometric and electronic structure was found to converge rapidly with slab thickness. Ionic charges were determined using a Mulliken partition of the total charge density [16]. The charges of the internal layers of the slab have returned to bulklike values and the field across the slab is zero for the $\mathrm{S} 4$, and thicker, slabs.

The converged electronic structure of the slab reveals a significant charge transfer from the (0001)-Zn to the (0001)-O surfaces of $0.17|e|$ which lowers the ionicity of both surfaces. On the basis of electrostatics, Noguera has previously predicted that such a charge transfer will stabilize the polar surfaces of $\mathrm{ZnO}$ [3]. The change in ionicity is highly localized in the surface region and has decayed to zero by the third bilayer below the surface plane. The computed charge transfer is coupled to the displacement of the atoms and hence phonon modes of the surface may lead to oscillations in the charge transfer between the (0001)-Zn and (0001)-O surfaces.

No unique surface energy can be determined for the (0001)-Zn and (0001)-O surfaces as cleavage of the bulk crystal creates both surfaces. Consequently, only the cleavage energy can be defined. The cleavage energy is computed to be $5.4 \mathrm{~J} \mathrm{~m}^{2}$ for the $\mathrm{S} 7 \mathrm{slab}$. Extrapolating its decay using an exponential function it converges to $\sim 4 \mathrm{~J} / \mathrm{m}^{2}$ for an infinitely thick slab. The efficiency of the electronic stabilization of the polar surface can be appreciated by noting that this is of the same order as the $2.32 \mathrm{~J} / \mathrm{m}^{2}$ required to cleave the (1010) surface [14]. Based on these energetics, one would expect that a polycrystalline sample of $\mathrm{ZnO}$ in thermal equilibrium to present a significant area of polar surface. This is confirmed by earlier temperature programmed desorption data [17] which also predicted that a significant area of the polar surface would be exposed in polycrystalline samples.

The geometric and electronic relaxations of the polar surface are strongly coupled. Consequently, it is very instructive to compare the computed relaxations to those deduced from high quality surface $\mathrm{x}$-ray diffraction studies. The relaxations of the $(000 \overline{1})-\mathrm{O}$ surface at room temperature were studied on station ID3BL7 of the European Synchrotron Radiation Facility (ESRF) [18]. X rays of $16.932 \mathrm{keV}$ were employed and the base pressure of the end station was $1 \times 10^{-10}$ mbar. The $\mathrm{ZnO}(000 \overline{1})-\mathrm{O}$ sample (Pi-kem) was prepared in situ by repeated cycles of $\mathrm{Ar}^{+}$sputtering and annealing at $1200 \mathrm{~K}$. Surface contamination was below the level of detection of Auger electron spectroscopy. Measurements were taken over a range of perpendicular momentum transfer $(l)$ for a given integer $(h, k)$ yielding crystal truncation rod (CTR) profiles [19]. In other respects, the measurements followed the methodology described by Charlton et al. [20]. Line scans were recorded along $(h, h),(h, 0)$, and $(0, k)$ at a fixed 1 value to examine possible evidence of a surface reconstruction, none being detected. Five CTRs were measured corresponding to $(h, k)=(2,0),(0,2),(1,1),(1,0)$, and $(0,1)$. The CTRs are presented in Fig. 2, where the structure factor is plotted against $l$, with the best fit data superimposed as a solid line. The data were fitted using a residual minimization method [21]. A total of 12 fitting parameters were used. Of these, 4 were displacements describing the vertical movements of the ions in the top two bilayers and 8 were Debye-Waller parameters describing their thermal vibrations. The scale factor and the fraction of the surface that adopts the fitted model were assumed to be $100 \%$.

The computed relaxations are compared to those deduced from our surface x-ray diffraction data in Table I. The very close agreement between theory and experiment for the $(000 \overline{1})-\mathrm{O}$ surface strongly supports our description of the electronic mechanism operating in zincite. The relaxations reported here are qualitatively similar to those recently derived from a more limited SXRD data set [22]. We note that the contraction in the first interlayer spacing 


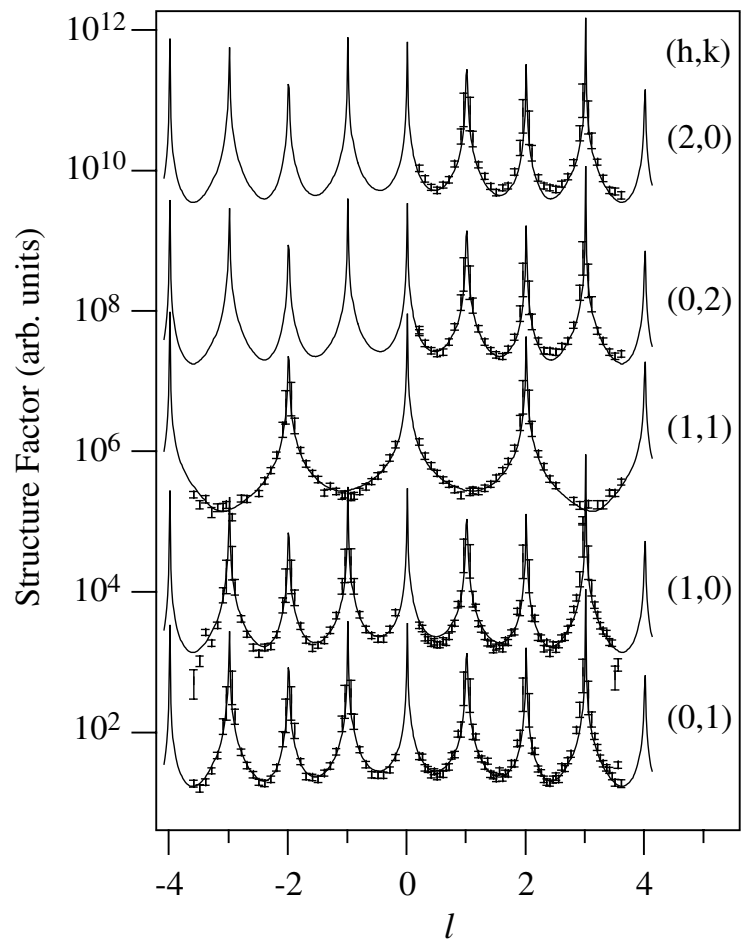

FIG. 2. Experimental CTR datapoints displayed on a $\log _{10}$ scale. The fitted CTR profiles are shown as solid curves through the datapoints, shown offset along the $y$ axis for clarity.

of the (0001)-Zn surface $(0.14 \AA)$ is considerably smaller than that of the $(000 \overline{1})$-O surface $(0.25 \AA)$. This is in line with one recent ion scattering experiment on the (0001)-Zn surface in which a contraction of the first to second layer spacing by $0.2 \AA$ was proposed on the basis of theoretical modeling [23], but is at variance with a further ion scattering study that predicted an expansion of the first to second layer distance by about $7 \%$ of the lattice spacing $(0.35 \AA)$, but did not consider movements of the second layer oxygen ions [24].

The computed band structure of the polar surfaces is shown in Fig. 3, which reveals further details of the stabilization mechanism. Both the (0001)-Zn and (0001)-O surfaces are predicted to be metallic, but the metallic nature of the surfaces arises from different causes. The charge trans-

TABLE I. Reconstructions of the $\mathrm{ZnO}(0001)$ and (0001) surfaces. A negative value is a movement towards the bulk of the material. All values are given in $\AA$, and the subscripts refer to bilayers numbered from the surface plane (e.g., $\mathrm{Zn}_{1}$ and $\mathrm{O}_{1}$ are the $\mathrm{Zn}$ and $\mathrm{O}$ ions in the surface bilayer).

\begin{tabular}{ccc}
\hline \hline Layer & $\mathrm{S} 7$ & SXRD \\
\hline$(0001)-\mathrm{Zn}_{1}$ & -0.07 & \\
$(0001)-\mathrm{O}_{1}$ & +0.07 & \\
$(000 \overline{1})-\mathrm{O}_{1}$ & -0.19 & $-0.22 \pm 0.05$ \\
$(000 \overline{1})-\mathrm{Zn}_{1}$ & +0.06 & $+0.016 \pm 0.008$ \\
$(000 \overline{1})-\mathrm{O}_{2}$ & & $-0.02 \pm 0.04$ \\
$(000 \overline{1})-\mathrm{Zn}_{2}$ & & $+0.010 \pm 0.007$ \\
\hline \hline
\end{tabular}

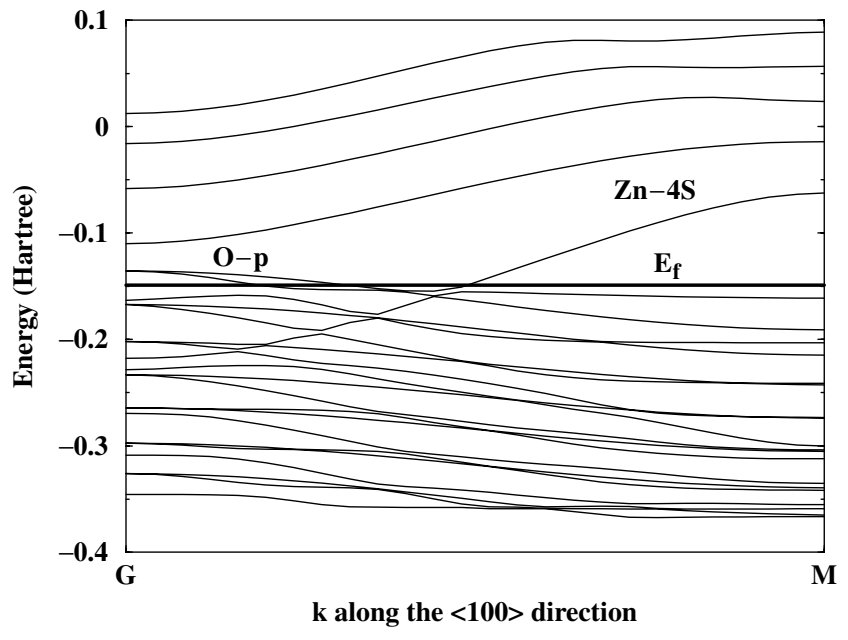

FIG. 3. The band structure of the S7 slab. The energy scale is in hartrees $(\mathrm{H})$. The partially occupied $\mathrm{Zn}-4 s$ band and the unoccupied O- $p$ band are labeled. $E_{f}$ denotes the Fermi level of the system.

ferred to the (0001)-Zn surface occupies the strongly dispersing $4 s$ band of the $\mathrm{Zn}$ ions in the surface layer - there is almost no occupancy of the $4 s$ states in subsurface layers. This charge has been extracted from a relatively narrow band localized in the surface layer of the (0001)-O surface of predominantly oxygen $p$ character but with a substantial admixture of $\mathrm{Zn}-d$ levels. Consequently, we predict that two-dimensional metallic layers are present at both surfaces. These features should be observable in angular resolved photoemission, but to our knowledge spectra from the basal faces have not been reported.

For the first time, $a b$ initio total energy calculations have been performed on the polar surfaces of an oxide material without artificially removing the macroscopic electric field. Instead, the electronic structure has been allowed to relax in the presence of the field. For the polar surfaces of zincite this results in significant charge transfer between the surfaces giving rise to remarkably stable surface structures. Surface $\mathrm{x}$-ray diffraction has been used to deduce the relaxations of the $(000 \overline{1})$-O surface which are in excellent agreement with those computed. The charge transfer leads to metallic surface layers which has obvious implications in gas sensing applications. Polar oxide surfaces are of growing technological importance in a wide range of applications due to their particular properties. These range through such diverse applications as catalysis [25], the newest giant magnetoresistive devices [26] (based on the polar (111) surface of $\mathrm{NiO}$ ), and in a variety of semiconductor devices [27]. The results and methodology of the current study are therefore of significance in a wide range of applications.

[1] N. F. Mott and R. W. Gurney, Electronic Processes in Ionic Crystals (Oxford University Press, Oxford, 1946). 
[2] P. A. Cox, Transition Metal Oxides: An Introduction to Their Electronic Structure and Properties (Clarendon Press, Oxford, 1992).

[3] See, for example, C. Noguera, Physics and Chemistry at Oxide Surface (Cambridge University Press, Cambridge, 1996).

[4] V.E. Henrich and P. A. Cox, The Surface Science of Metal Oxides (Cambridge University Press, Cambridge, 1994).

[5] P. W. Tasker, J. Phys. C 12, 4977 (1979).

[6] A. Pojani, F. Finocchi, and C. Noguera, Appl. Surf. Sci. 142, 177 (1999).

[7] A. Pojani, F. Finocchi, and C. Noguera, Surf. Sci. 442, 179 (1999).

[8] W. Hebenstreit, M. Schmid, J. Redinger, R. Podloucky, and P. Varga, Phys. Rev. Lett. 85, 5376 (2000).

[9] T. M. Parker, N. G. Condon, R. Lindsay, F. M. Leibsle, and G. Thornton, Surf. Sci. 415, L1046 (1998).

[10] A. D. Becke, J. Chem. Phys. 98, 5648 (1993).

[11] C. Lee, W. Yang, and R. G. Parr, Phys. Rev. B 37, 785 (1988).

[12] R. Dovesi, C. Roetti, M. Causà, N. M. Harrison, R. Orlando, and E. Aprà, CRYSTAL98 Users's Manual (University of Torino, Torino, 1996).

[13] J. Muscat, A. Wander, and N. M. Harrison, Phys. Rev. Lett. (to be published).

[14] A. Wander and N. M. Harrison, Surf. Sci. (to be published).
[15] E.g., S. Mankefors, Phys. Rev. B 59, 13151 (1999).

[16] C. Pisani, R. Dovesi, and C. Roetti, Hartree-Fock ab initio Treatment of Crystalline Systems, Lecture Notes in Chemistry (Springer-Verlag, Heidelberg, 1988), Vol. 48.

[17] M. Bowker, H. Houghton, K. C. Waugh, T. Giddings, and M. Green, J. Catal. 84, 252 (1983)

[18] S. Ferrer and F. Comin, Rev. Sci. Instrum. 66, 1674 (1995).

[19] R. Feidenhans, Surf. Sci. Rep. 10, 105 (1989).

[20] G. Charlton, P. B. Howes, C. L. Nicklin, P. Steadman, J. S. G. Taylor, C. A. Muryn, S.P. Harte, J. Mercer, R. McGrath, D. Norman, T.S. Turner, and G. Thornton, Phys. Rev. Lett. 78, 495 (1997).

[21] E. Vlieg, J. Appl. Crystallogr. (to be published).

[22] N. Jedrecy, M. Sauvage-Simkin, and R. Pinchaux, Appl. Surf. Sci. (to be published).

[23] S. H. Overbury, P. V. Radulovic, S. Thevuthasan, G. S. Herman, M. A. Henderson, and C. H. F. Pedin, Surf. Sci. 410, 106 (1998).

[24] H. Maki, N. Ichinose, N. Ohashi, H. Haneda, and J. Tanaka, Surf. Sci. 457, 377 (2000).

[25] D. Cappus et al., Chem. Phys. 177, 533 (1993).

[26] S. Soeya, S. Nakamura, T. Imagawa, and S. Narishige, J. Appl. Phys. 77, 5838 (1995).

[27] S. Makefors, P. O. Nilsson, and J. Kanski, Surf. Sci. 443, L1049 (1999). 